\title{
Albedo da superfície por meio de imagens TM-Landsat 5 e modelo numérico do terreno
}

\author{
Pedro R. Giongo ${ }^{1} \&$ Carlos A. Vettorazzi ${ }^{2}$ \\ ${ }^{1}$ UEG. Santa Helena de Goias, GO. E-mail: pedro.giongo@ueg.br (Autor correspondente) \\ ${ }^{2}$ ESALQ/USP. Piracicaba, SP. E-mail: cavettor@usp.br
}

Palavras-chave:

bacia hidrográfica

sensoriamento remoto

refletividade

METRIC

\begin{abstract}
R E S U M O
Objetivou-se, na presente pesquisa, estimar o albedo da superfície na Bacia do Rio Corumbataí, localizada no Estado de São Paulo, Brasil, com uso de imagens do Mapeador Temático (TM) do satélite Landsat 5, juntamente com o Modelo Numérico do Terreno (MNT) para considerar a inclinação e o sentido de declive do terreno, além da aplicação do algoritmo Mapping Evapotranspiration at High Resolution and with Internalized Calibration (METRIC). Utilizaram-se dados complementares disponíveis em estação meteorológica localizada na região de estudo e dez imagens datadas de 01/02/2009, 04/02/2010, 22/04/2009, 24/05/2009, 12/06/2010, 08/07/2008, 30/07/2010, 31/08/2010, 13/09/2009 e 31/10/2009. O uso de imagens TM-Landsat 5 com o algoritmo METRIC e o MNT permitiu identificar as variações espaciais e temporais de diferentes coberturas no mapeamento do albedo da superfície da Bacia do Rio Corumbataí. As avaliações foram consistentes com os valores obtidos na literatura para os alvos cana-de-açúcar, água, pastagem e eucalipto, nas imagens em dias de céu claro.
\end{abstract}

Key words: river basin remote sensing reflectivity METRIC

\section{Surface albedo through TM-Landsat 5 images and Digital Terrain Model}

\begin{abstract}
A B S T R A C T
This study aimed at to estimate the surface albedo in the Corumbataí River Basin, State of São Paulo, Brazil, using TM-Landsat 5 images, together with the Digital Terrain Model (DTM), in order to take into account the terrain slope, as well as the Mapping Evapotranspiration at High Resolution and with Internalized Calibration (METRIC) model. Available ancillary data from a meteorological station located in the study area, and ten images from 01/02/2009, 04/02/2010, 22/04/2009, 24/05/2009, 12/06/2010, 08/07/2008, 30/07/2010, 31/08/2010, 13/09/2009 and 31/10/2009, were also used. The use of TM-LANDSAT 5 images with the METRIC model and the DTM, allowed to identify the spatial and temporal variations of different coverages on the mapping of surface albedo of the Corumbataí River Basin. The data were consistent with those presented in the literature for sugarcane, water, pasture and eucalypt, for satellite images of clear-sky days.
\end{abstract}

\section{INTRODUÇÃO}

O albedo da superfície é um parâmetro biofísico cuja variação ocorre com o tipo de alvo sobre o qual incide a radiação eletromagnética e variações deste parâmetro são excelentes indicadores de mudanças no cenário de uma bacia hidrográfica (Silva et al., 2005).

As constantes mudanças na cobertura e o uso da terra têm influenciado muito as alterações no comportamento dos componentes do balanço de energia na superfície terrestre, bem como todas as variáveis biofísicas. As variáveis biofísicas são importantes e influenciadas pela alteração da cobertura vegetal e servem de estudo como indicadores dos padrões de mudança do clima de uma região, como observado no estudo de Loarie et al. (2011).

De acordo com Bastiaanssen (2000) e Menezes et al. (2009) o sensoriamento remoto aplicado à agricultura favorece a obtenção de informações possibilitando a geração de séries temporais em larga escala de espaço. Mencionada ferramenta tem sido fundamental para permitir a aplicação de algoritmos nesses estudos, como citaram Hafeez et al. (2002) que as técnicas de sensoriamento remoto para estimativas dos componentes do balanço de energia e da evapotranspiração, com larga cobertura espacial, são utilizadas desde 1990.

Diferentes algoritmos têm surgido nos últimos anos para o mapeamento das variáveis biofísicas, dos componentes do balanço de energia e evapotranspiração, sendo o Mapping Evapotranspiration at High Resolution and with Internalized Calibration (METRIC) um dos mais aplicados e com ótimos resultados de desempenho nos mais diferentes tipos de superfície e condições, conforme trabalhos de Allen et al. (2007a, b) e Tasumi et al. (2008).

No algoritmo METRIC o albedo da superfície é obtido a partir da reflectância, corrigidos os efeitos atmosféricos banda 
a banda e se estimando o cômputo da reflectância da superfície para cada banda do sensor cuja transmissividade é obtida como função da pressão atmosférica e do vapor d'água presente na atmosfera. Esta metodologia foi testada por Silva et al. (2008) os quais constataram que o METRIC apresentou grande precisão confirmada por comparação com medidas piranométricas terrestres, com erro padrão da estimativa de apenas 0,01 .

O METRIC, apresentado por Allen et al. (2005) e Allen et al. (2007a, b), representa um aprimoramento do Surface Energy Balance Algorithm for Land (SEBAL) e vem sendo aplicado com sucesso a superfícies heterogêneas e áreas montanhosas, adotando-se procedimentos que envolvem o processamento de imagens espectrais requeridas no SEBAL e a inclusão de Modelo Numérico do Terreno (MNT). Segundo Souza et al. (2011) a aplicação do MNT nas estimativas de componentes de energia através de imagens de satélite, melhora os resultados uma vez que ele corrige a inclinação e o sentido da rampa do terreno.

Como importante fator para avaliar as mudanças de cobertura, o albedo é necessário para obter o saldo de radiação em superfície. Em grandes áreas essas informações só serão possíveis se forem utilizadas imagens de satélite visto estas terem permitido inúmeros trabalhos espacializando a informação de forma rápida e confiável (Giongo et al.,2010; Moreira et al., 2010).

Neste contexto o objetivo do trabalho foi avaliar a viabilidade do uso de imagens TM-Landsat 5, Modelo Numérico do Terreno e o algoritmo METRIC, no mapeamento do albedo da superfície na bacia do Rio Corumbataí, Estado de São Paulo e avaliar, ainda, as estimativas de albedo obtidas em cana-de-açúcar, água, cerrado, pastagem e eucalipto.

\section{Material e Métodos}

A Bacia do Rio Corumbataí, localizada na porção centroleste do Estado de São Paulo entre os paralelos 22 $04^{\prime} 46^{\prime \prime} \mathrm{S}$ e $22^{\circ} 41^{\prime} 28^{\prime \prime} \mathrm{S}$ e os meridianos $47^{\circ} 27^{\prime} 40^{\prime \prime} \mathrm{W}$ e $47^{\circ} 55^{\prime} 03^{\prime \prime} \mathrm{W}$, tem aproximadamente 170.000 ha. O valor aproximado de perímetro é $301,52 \mathrm{~km}$, tendo $63,72 \mathrm{~km}$ de extensão na direção norte-sul e 50,65 km na direção leste-oeste. É uma sub-bacia do Rio Piracicaba, situada na sua margem direita.

O clima da região, na classificação de Köppen, é do tipo Cwa, ou seja, subtropical, seco no inverno e chuvoso no verão, com temperatura média do mês mais quente superior a $22^{\circ} \mathrm{Ce}$ precipitação anual em torno de $1390 \mathrm{~mm}$ (Salati, 1996).

A Figura 1 indica a localização da Bacia Hidrográfica do Rio Corumbataí, SP, além de alguns alvos de áreas homogêneas em sua superfície, com a localização geográfica: Estação Meteorológica, $22^{\circ} 42^{\prime}$ 09,14" S e 470 37' 18,65” W; cana-deaçúcar, $22^{\circ} 26^{\prime} 20,35^{\prime \prime} \mathrm{S}$ e $47^{\circ} 37^{\prime} 39,08^{\prime \prime}$ W; pastagem, $22^{\circ} 13^{\prime}$

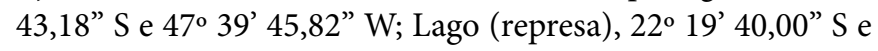
$47^{\circ} 29^{\prime} 36,90^{\prime \prime}$ W; e eucalipto, $22^{\circ} 13^{\prime} 27,70^{\prime \prime}$ 'S e $47^{\circ} 44^{\prime} 10,00^{\prime \prime}$ W.

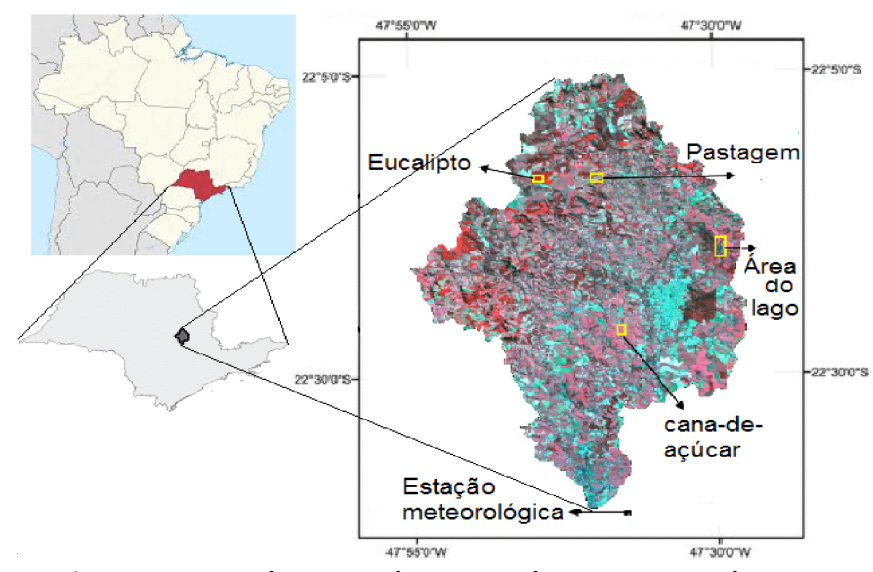

Figura 1. Localização da Bacia do Rio Corumbataí e imagem TM-Landsat 5, composição colorida RGB432, de 08 de julho de 2008, com a localização dos alvos estudados

Referidos alvos foram estudados para obter informações dos pixels nas imagens em áreas homogêneas. A estação meteorológica automática com coleta de dados a cada $15 \mathrm{~min}$ está localizada na área Experimental da ESALQ/USP e registra os dados de: temperatura do ar, umidade relativa, radiação ascendente e descendente, velocidade e direção do vento, pressão de vapor, precipitação.

Selecionaram-se as imagens TM-Landsat 5 (órbita 220, pontos 75 e 76) considerando-se a cobertura espacial total da bacia do Rio Corumbataí, a distribuição temporal que permitisse estudar os diferentes períodos do ano e ainda que houvesse nenhuma ou o mínimo de cobertura de nuvens sobre a área de estudo (Tabela 1). As imagens foram obtidas na Divisão de Geração de Imagens (DGI) do Instituto Nacional de Pesquisas Espaciais (INPE) processadas pelo software Erdas Imagine 9.1.

O Modelo Numérico do Terreno (MNT) da área de estudo foi obtido com resolução espacial de $90 \times 90 \mathrm{~m}$, referente ao mosaico x (27) e y (17) (SRTM, 2009). Efetuou-se a reamostragem dos pixels para resolução espacial de $30 \times 30$ $\mathrm{m}$ proporcionando, assim, a compatibilidade com as imagens TM-Landsat 5.

A primeira etapa do trabalho foi constituída pelo cômputo da radiância espectral de cada banda $\left(L_{\lambda_{i}}\right)$, ou seja, a efetivação da calibração radiométrica em que o nível de cinza de cada pixel da imagem é convertido em radiância espectral monocromática. Essas radiâncias representam a energia solar refletida por cada pixel, por unidade de área, de tempo, de ângulo sólido e de comprimento de onda medida a nível do satélite Landsat (705 $\mathrm{km}$ ) para as bandas 1, 2, 3, 4, 5 e 7 (Markham \& Baker,1987).

$$
\mathrm{L}_{\lambda \mathrm{i}}=\mathrm{a}_{\mathrm{i}}+\frac{\mathrm{b}_{\mathrm{i}}-\mathrm{a}_{\mathrm{i}}}{255} \mathrm{NC}
$$

em que:

Tabela 1. Imagens TM-Landsat 5 , com a data de aquisição da imagem (DAI) e o dia sequencial do ano (DS), com cobertura da órbita 220, pontos 75 e 76

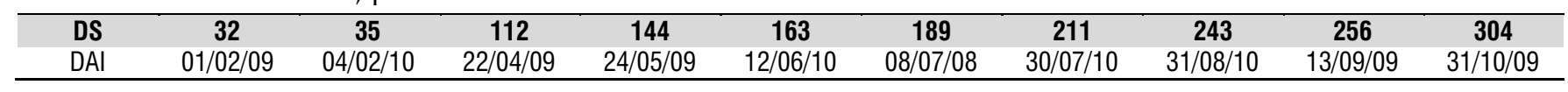


a e b - radiâncias espectrais mínimas e máximas, $\mathrm{Wm}^{-2} \mathrm{sr}^{-1}$ $\mu \mathrm{m}^{-1}$, Tabela 2

NC - nível de cinza de cada banda

i - bandas $(1,2, \ldots$ e 7$)$ do satélite Landsat-5, sensor TM

Tabela 2. Descrição das bandas do TM-Landsat 5, com os intervalos correspondentes de comprimento de onda $(\lambda)$, coeficientes de calibração (a e b, radiâncias mínima e máxima, respectivamente) e irradiâncias espectrais no topo da atmosfera (Toa)

\begin{tabular}{ccccc}
\hline Banda & $\boldsymbol{\lambda}(\boldsymbol{\mu m})$ & $\mathbf{a}$ & $\mathbf{b}$ & $\mathbf{T o a}\left(\mathbf{W m}^{-2} \boldsymbol{\mu m}^{-1}\right)$ \\
1 & $0,45-0,52$ & $-1,52$ & 169 & 1983 \\
2 & $0,52-0,60$ & $-2,84$ & 333 & 1796 \\
3 & $0,63-0,69$ & $-1,17$ & 264 & 1536 \\
4 & $0,76-0,90$ & $-1,51$ & 221 & 1031 \\
5 & $1,55-1,75$ & $-0,37$ & 30,2 & 220 \\
6 & $10,45-12,42$ & 1,2378 & 15,3032 & - \\
7 & $2,08-2,35$ & $-0,15$ & 16,5 & 83,44 \\
\hline
\end{tabular}

Fonte: Chander et al. (2009)

A reflectância monocromática de cada banda $\left(\rho_{\mathrm{aa}}\right)$ é definida como sendo a razão entre o fluxo de radiação refletida e o fluxo de radiação incidente, obtida segundo a equação proposta por Allen et al. (2002):

$$
\rho_{\mathrm{ai}}=\frac{\pi \mathrm{L}_{\lambda \mathrm{i}}}{\mathrm{k}_{\lambda \mathrm{i}} \cdot \cos \theta \cdot \mathrm{dr}}
$$

em que:

$\mathrm{L}_{\lambda \mathrm{i}} \quad$ - radiância espectral de cada banda, $\mathrm{Wm}^{-2} \mathrm{sr}^{-1} \mu \mathrm{m}^{-1}$

$\mathrm{k}_{\lambda \mathrm{i}} \quad$ - irradiância solar espectral, banda $i$ no topo da atmosfera, $\mathrm{Wm}^{-2} \mathrm{sr}^{-1} \mu \mathrm{m}^{-1}$, Tabela 2

$\mathrm{dr} \quad$ - inverso do quadrado da distância relativa Terra-Sol (em unidade astronômica - UA), dada por Iqbal (1983):

$$
\mathrm{dr}=1+0,033 \cos \left(\frac{\mathrm{DS} 2 \pi}{365}\right)
$$

$\theta \quad$ - ângulo de incidência da radiação solar que é obtido em cada pixel mediante uso de MNT e equação proposta por Duffie \& Beckman (1991):

$$
\begin{aligned}
\cos \theta & =\operatorname{sen}(\delta) \operatorname{sen}(\phi) \cos (\mathrm{s})-\operatorname{sen}(\delta) \cos (\phi) \operatorname{sen}(\mathrm{s}) \cos (\gamma)+ \\
& +\cos (\delta) \cos (\phi) \cos (\mathrm{s}) \cos (\omega)+\cos (\delta) \operatorname{sen}(\phi) \operatorname{sen}(\mathrm{s}) \cos (\gamma) \cos (\omega)+ \\
& +\cos (\delta) \operatorname{sen}(\mathrm{s}) \operatorname{sen}(\gamma) \operatorname{sen}(\omega)
\end{aligned}
$$

em que:

$\delta \quad$ - declinação do sol

$\varphi \quad$ - latitude do pixel (positiva no Hemisfério Norte e negativa no Hemisfério Sul)

$s \quad$ - inclinação da superfície, sendo $s=0$ para superfície horizontal e $s=\pi / 2 \mathrm{rad}$ para inclinação vertical

(s é sempre positivo e representa a inclinação em qualquer direção)

$\gamma \quad$ - ângulo do aspecto da superfície em que $\gamma=0$ para inclinação orientada para o Sul, $\gamma=-\pi / 2 \operatorname{rad}$ para inclinação orientada para o Leste, $\gamma=+\pi / 2$ rad para inclinação voltada para o Oeste e $\gamma= \pm \pi$ rad para inclinações orientadas para o Norte

$\omega$ - ângulo horário do sol com $\omega=0$ ao meio-dia solar, negativo antes do meio-dia e positivo depois do meio-dia

As faixas espectrais delimitadas entre os limites superior $\left(\mathrm{UP}_{\mathrm{i}}\right)$ e inferior $\left(\mathrm{LO}_{\mathrm{i}}\right)$ (Tabela 3 ) também incluem as regiões espectrais não sensoriadas pelo TM-Landsat 5 , dentro do intervalo de comprimento de onda curtas de 0,3 a 4,0 $\mu \mathrm{m}$. Segundo Tasumi et al. (2008) a inclusão dessas regiões espectrais no cálculo dos fatores de ponderação permite definir melhor a contribuição relativa de cada banda espectral para a estimativa do albedo da superfície.

Após transformar os valores de reflectância aparente $\left(\rho_{\mathrm{a}}\right)$ para reflectância atmosférica corrigida $\left(\rho_{c}\right)$ é necessário estimar os efeitos de espalhamento e absorção da radiação pela atmosfera, através da Eq. 5 (Tasumi et al., 2008).

$$
\rho_{\mathrm{s}, \mathrm{i}}=\frac{\rho_{\mathrm{a}, \mathrm{i}}-\rho_{\mathrm{t}, \mathrm{i}}}{\tau_{\mathrm{in}, \mathrm{i}} \cdot \tau_{\mathrm{out}, \mathrm{i}}}
$$

em que:

$\rho_{s, i}$ - reflectância espectral corrigida dos efeitos atmosféricos na banda $i$

$\rho_{\mathrm{a}, \mathrm{i}} \quad$ - reflectância espectral aparente na banda i no topo da atmosfera (Eq. 2)

$\rho_{\mathrm{t}, \mathrm{i}} \quad$ - reflectância atmosférica (radiação difusa da atmosfera ao sensor) na banda $\mathrm{i}$

$\tau_{\text {in,i }}$ e $\tau_{\text {out,i }}$ - valores da transmitância da atmosfera relativa à radiação solar incidente e refletida pela superfície, respectivamente, para cada banda $\mathrm{i}$

A estimativa da transmitância atmosférica nas duas direções é determinada a partir do conhecimento da umidade relativa do ar no momento da passagem do satélite sobre a área de estudo monitorada na estação meteorológica de superfície. A transmitância da atmosfera relativa à radiação solar incidente na superfície $\left(\mathrm{t}_{\mathrm{in}}\right)$ proposta por Tasumi et al. (2008):

$$
\tau_{\mathrm{in}, \mathrm{i}}=\mathrm{c}_{1, \mathrm{i}} \cdot \exp \left[\frac{\mathrm{c}_{2, \mathrm{i}} \mathrm{P}_{\mathrm{ar}}-\mathrm{c}_{3, \mathrm{i}} \mathrm{W}-\mathrm{c}_{4, \mathrm{i}}}{\cos \theta}\right]+\mathrm{c}_{5, \mathrm{i}}
$$

\begin{tabular}{|c|c|c|c|c|c|c|}
\hline Banda & 1 & 2 & 3 & 4 & 5 & 7 \\
\hline Faixa espectral dos limites UP ${ }_{i}$ e $\mathrm{LO}_{\mathrm{i}}(\mu \mathrm{m})$ & 0,300 a 0,520 & 0,520 a 0,615 & 0,615 a 0,725 & 0,725 a 1,225 & 1,225 a 1,915 & 1,915 a 4,000 \\
\hline Peso $\mathrm{w}_{\mathrm{i}}$ & 0,254 & 0,149 & 0,147 & 0,311 & 0,103 & 0,036 \\
\hline
\end{tabular}

em que:

$\mathrm{P}_{\mathrm{ar}}$ - pressão atmosférica $(\mathrm{kPa})$ calculada pela Eq. 7 (EWRI, 2005)

Tabela 3. Faixas espectrais das bandas do TM compreendidas entre os limites superior $\left(\right.$ UP $\left._{i}\right)$ e inferior $\left(L_{i}\right)$ e, respectivos fatores de ponderação (peso $\mathrm{w}_{\mathrm{i}}$ )

Fonte: Tasumi et al. (2008) 
$\theta \quad$ - ângulo de incidência da radiação, calculado pela Eq. 4

$\mathrm{W}$ - água precipitável na atmosfera $(\mathrm{mm})$ calculada pela Eq. 8

$\mathrm{c}_{1, \mathrm{i}}$ a $\mathrm{c}_{5, \mathrm{i}}$ - parâmetros constantes determinados para cada banda i, por Tasumi et al. (2008) mediante o modelo de transferência radiativa Simple Model of Atmospheric Radiative Transfer of Sunshine (SMARTS2) (Tabela 4):

$$
\mathrm{P}_{\mathrm{ar}}=101,3\left(\frac{\mathrm{T}_{\mathrm{ar}}-0,0065 \mathrm{~h}}{\mathrm{~T}_{\mathrm{ar}}}\right)^{5,26}
$$

em que:

$\mathrm{T}_{\mathrm{ar}} \quad$ - temperatura do ar (K) obtida na estação meteorológica

$\mathrm{h}$ - altitude média da superfície em relação a nível médio do mar, $m$

W - função da quantidade de água precipitável em um ponto da imagem (local da estação meteorológica) calculada pela equação proposta por Garrison \& Adler (1990):

$$
\mathrm{W}=0,14 \mathrm{e}_{\mathrm{a}} \mathrm{P}_{\mathrm{ar}}+2,1
$$

em que:

$\mathrm{e}_{\mathrm{a}} \quad$ - pressão de vapor de água $(\mathrm{kPa})$ e $\mathrm{W}, \mathrm{mm}$

A transmitância relativa à radiação solar refletida pela superfície $\left(\tau_{\text {out }}\right)$ foi determinada por Tasumi et al. (2008):

$$
\tau_{\text {out }, \mathrm{i}}=\mathrm{c}_{1, \mathrm{i}} \cdot \exp \left[\frac{\mathrm{c}_{2, \mathrm{i}} \mathrm{P}_{\mathrm{ar}}-\mathrm{c}_{3, \mathrm{i}} \mathrm{W}-\mathrm{c}_{4, \mathrm{i}}}{\cos \eta}\right]+\mathrm{c}_{5, \mathrm{i}}
$$

em que:

$\eta \quad$ - ângulo de visada do sensor TM (próximo de $0 \mathrm{e}$, portanto, $\cos \eta=1$ ).

Após determinar a transmitância atmosférica nas duas direções, Tasumi et al. (2008) procederam ao cálculo da reflectância atmosférica $\left(\rho_{\mathrm{t}, \mathrm{i}}\right)$ para cada banda do TM através da inversão da Eq. 6 e do uso do dado MOD02 (radiância do MODIS) definindo então os parâmetros $C_{b}$, para uso na Eq. 10 .

$$
\rho_{\mathrm{t}, \mathrm{i}}=\mathrm{Cb}\left(1-\tau_{\mathrm{in, \textrm {i }}}\right)
$$

em que:

$\mathrm{C}_{\mathrm{b}}$ - parâmetro determinado para cada banda i do sensor TM (Tabela 4)
Finalmente, o albedo de superfície é obtido com a Eq. 11 (Tasumi et al., 2008).

$$
\alpha=\sum_{\mathrm{i}=1}^{6}\left(\rho_{\mathrm{s}, \mathrm{i}} \mathrm{w}_{\mathrm{i}}\right)
$$

em que:

$$
\begin{aligned}
& \rho_{\mathrm{s}, \mathrm{i}} \quad \text { - reflectância espectral corrigida } \\
& \mathrm{w}_{\mathrm{i}} \text { - fator de ponderação (Tabela } 3 \text { ). }
\end{aligned}
$$

\section{Resultados e Discussão}

Verificou-se que os valores de albedo da superfície na parte leste da bacia foram pouco inferiores aos da parte oeste e tais diferenças podem ocorrer pela predominância de alguns tipos de cobertura da superfície. De modo geral, a parte leste apresenta relevo menos montanhoso propiciando, assim, maior quantidade de áreas agricultáveis, como culturas de cana-deaçúcar, que apresentam predominância de albedo inferior $(0,13$ a 0,25 ) comparado ao da pastagem (Figura 2).

A heterogeneidade na cobertura da superfície é a principal razão da variação de albedo dentro da bacia, além de considerável variação da disponibilidade hídrica durante o período estudado. Querino et al. (2006) citaram haver grande variação nos valores entre o período seco e o chuvoso, constatados neste estudo. Rodrigues et al. (2009) também verificaram que as mudanças de vigor dos vegetais, que são mais verdes no período chuvoso

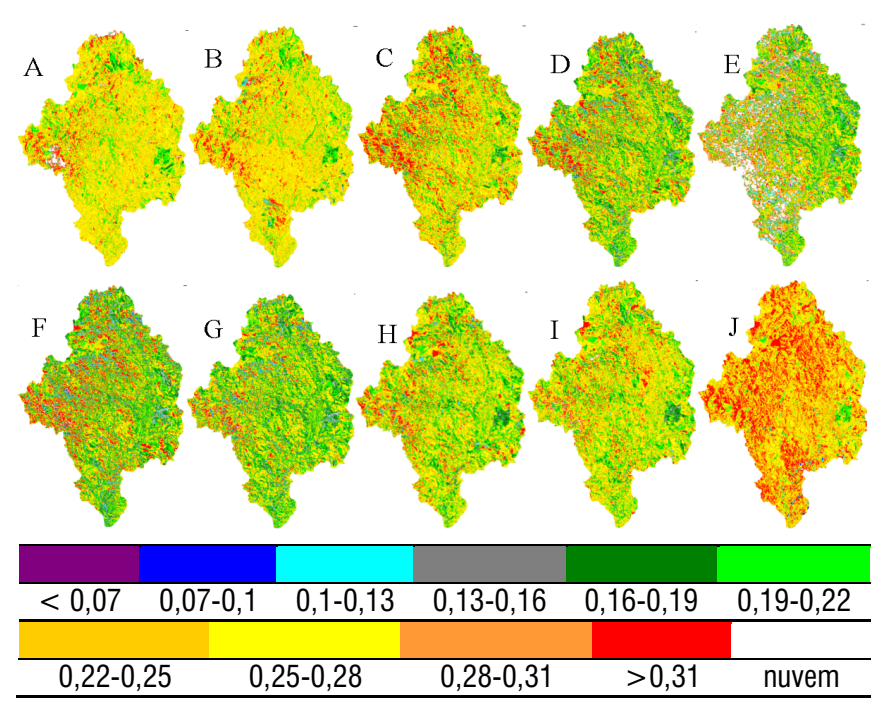

Figura 2. Imagens de albedo nos dias sequencial do ano (DS) 32 (A), 35 (B), 112 (C), 144 (D), 163 (E), 189 (F), 211 (G), 243 (H), 256 (I) e 304 (J) da Bacia do Rio Corumbataí, SP

Tabela 4. Coeficiente de calibração $C_{1}$ a $C_{5}$ e $C_{b^{\prime}}$ para as bandas 1, 2, 3, 4, 5 e 7 do TM-Landsat 5

\begin{tabular}{ccccccc}
\hline \multirow{2}{*}{ Coeficientes } & \multicolumn{7}{c}{ Bandas } \\
\cline { 2 - 8 } $\mathrm{C}_{1}$ & $\mathbf{1}$ & $\mathbf{2}$ & $\mathbf{3}$ & $\mathbf{4}$ & $\mathbf{5}$ & $\mathbf{7}$ \\
$\mathrm{C}_{2}$ & 0,987 & 2,319 & 0,951 & 0,375 & 0,234 & 0,365 \\
$\mathrm{C}_{3}$ & $-0,00071$ & $-0,00016$ & $-0,00033$ & $-0,00048$ & $-0,00101$ & $-0,00097$ \\
$\mathrm{C}_{4}$ & 0,000036 & 0,000105 & 0,00028 & 0,005018 & 0,004336 & 0,004296 \\
$\mathrm{C}_{5}$ & 0,0880 & 0,0437 & 0,0875 & 0,1355 & 0,0560 & 0,0155 \\
$\mathrm{C}_{\mathrm{b}}$ & 0,0789 & $-1,2697$ & 0,1014 & 0,6621 & 0,7757 & 0,639 \\
\hline
\end{tabular}


devido à presença da umidade, são fatores condicionantes das mudanças sazonais no albedo da superfície.

Em pequena porção da bacia os menores valores de albedo entre 6 e 11\% foram obtidos em superfícies de água, como represas e o próprio rio Corumbataí. Ainda Silva et al. (2005) obtiveram mínimos de 6,8 e 7,4\% para os anos de 2000 e 2001, respectivamente destacando que os valores mínimos foram obtidos no rio São Francisco concordando, portanto, com os obtidos nesta pesquisa.

Os valores máximos de albedo na bacia foram superiores a $31 \%$, com destaque para a Figura 2J, em que houve maior percentual da bacia com valores entre 28 a $31 \%$. O final do período seco desta região contribuiu significativamente para elevar esses valores de albedo, assim como as áreas de baixa disponibilidade hídrica, que elevaram os valores de albedo em trabalho de Silva et al. (2011).

Os altos valores de albedo $(>0,31)$ foram obtidos em áreas específicas, com pouca (áreas de cerrado) ou nenhuma vegetação, além de áreas agricultáveis, que estavam com solo exposto. Valores elevados também foram observados por Silva et al. (2005) em que o albedo em solo exposto foi de 31 e 33\% para os anos de 2000 e 2001, respectivamente.

A média geral de albedo da superfície para as imagens foi de 20 e $30 \%$ (Figura 3) cujos principais condicionantes desta amplitude são as mudanças das características na cobertura vegetal e a disponibilidade hídrica do solo e da movimentação natural do Sol. Tais resultados condizem com Silva et al. (2005) com albedo variando de 20,5 e $21 \%$ nos anos de 2000 e 2001, respectivamente, na região do perímetro irrigado entre os estados da Bahia e Pernambuco.

Buscando avaliar melhor o desempenho da metodologia proposta para a determinação do albedo da superfície, realizaram-se recortes de áreas homogêneas dentro da cena estudada. Quatro recortes de diferentes alvos foram extraídos, com tamanho de aproximadamente 10 pixels, cujas áreas representam cana-de-açúcar, corpo d'água (uma represa) eucalipto e pasto (Figura 1).

É possível verificar que na área de cana-de-açúcar a amplitude do albedo em área homogênea foi de 26 a 29\%, enquanto os maiores valores de albedo foram registrados nas imagens do período de início e final de ano, como dos DS 304, 35, 256 (Figura 3); este comportamento anual também foi observado por Gomes et al. (2009) na região de Santa Rita do Passa Quatro, SP.

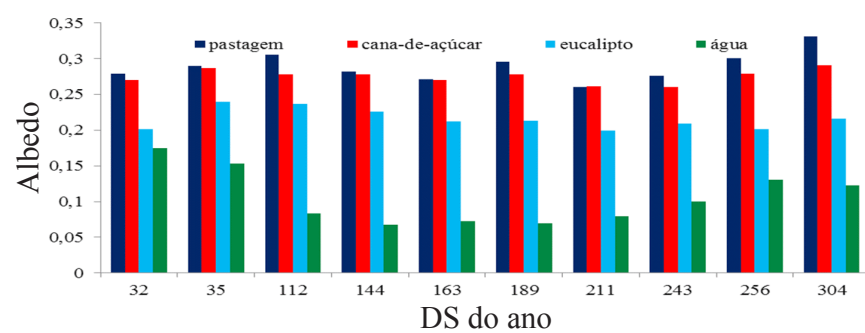

Figura 3. Média dos valores de albedo dos pixels nas áreas de cana-de-açúcar, represa, eucalipto e pastagem nos dias sequencial do ano (DS) 32, 35, 112, 144, 163, 189, 211, 243, b256 e 304 na Bacia do Rio Corumbataí, SP
Esses valores $(0,26$ a 0,29$)$ são superiores aos obtidos por Giongo et al. (2010), que obtiveram albedo em área de cana-deaçúcar com valores entre 13,5 a 23,1\%. Já Cabral et al. (2003) registraram valores entre 12 e $32 \%$ para a cana-de-açúcar não irrigada, dependendo da fase de crescimento da cultura, corroborando com os resultados obtidos neste trabalho para áreas de cana-de-açúcar.

Valores de albedo em corpo hídrico (represa) ficaram entre $5 \mathrm{e}$ $18 \%$, conforme a Figura 3. Observou-se que no período de verão há, quando ocorrem os eventos chuvosos, tendência de aumento do albedo pelo acúmulo de impurezas carregadas pelas enxurradas da chuva em direção à represa, deixando-a turva e não representando o albedo real de água limpa, Como observado por Rodrigues et al. (2009) ao constatarem os menores valores de albedo de superfície no reservatório Trussu, $\mathrm{CE}$, em períodos secos por não ocorrer a descarga de sedimentos advindos da ocorrência de chuvas; já nos demais períodos do ano foram registrados valores de albedo entre 5 e $11 \%$ e que estão de acordo com os obtidos por Giongo et al. (2010) que obtiveram 1,7 a 10,4\%, também Silva et al. (2005) com valores entre 7 e 9\%; Bastiaanssen (2000) obteve valores entre 3 a $10 \%$, todos em superfícies de água.

$\mathrm{Na}$ área plantada com eucalipto (Figura 3) o registro de albedo se mantém entre 19 e $25 \%$, sendo possível observar baixa amplitude dos valores, reflexo da pequena modificação em características por ser cultura perene e que podem ser comparadas a áreas de mata, ou vegetação densa, que apresentam comportamento semelhante. Ainda Silva et al. (2005) em estudo com o mesmo sensor e satélite e o algoritmo SEBAL, registraram albedo semelhante aos desta pesquisa, corroborando com os valores obtidos. Boegh et al. (2002) na Dinamarca obtiveram, com imagens TM-Landsat $5 \mathrm{em}$ áreas de vegetação densa, valor de $18 \%$ de albedo.

$\mathrm{Na}$ área de pastagem (Figura 3) registrou-se albedo entre 25 e $34 \%$, no período estudado, valores que corroboram com os obtidos por Iziomons \& Mayer (2002) que, avaliando o comportamento do albedo em áreas de pastagens no sudoeste da Alemanha, entre 1991 e 1996, constataram acentuada variação no período estudado com valores entre 22,2 a $36 \%$. A alta variação de albedo em áreas de pastagem é comum nesta região, consequência do volume e da distribuição das chuvas irregulares, o que pode ser observado nas Figuras 2 e 3 (DS 163 e 304).

\section{Conclusões}

1. Verifica-se boa consistência entre a literatura e os valores de albedo obtidos na Bacia do Rio Corumbataí, SP, com imagens TM-Landsat 5, o algoritmo METRIC e o MDT;

2. Há grande variação anual para albedo de superfície na água, enquanto áreas de cana-de-açúcar, eucalipto e pastagem, têm baixa variação anual;

\section{Literatura Citada}

Allen, R. G.; Tasumi, M.; Morse, A.; Trezza, R. A landsat-based energy balance and evapotranspiration model in Western US water rights regulation and planning. Irrigation and Drainage Systems, v.19, p.251-268, 2005. 
Allen, R. G.; Tasumi, M.; Morse, A.; Trezza, R.; Wright, J. L.; Bastiaanssen, W. G. M.; Kramber, W.; Lorite, I.; Robison, C. W. Satellite-based energy balance for mapping evapotranspiration with internalized calibration (METRIC) - Applications. Journal of Irrigation and Drainage Engineering, v.133, p.395-406, 2007a.

Allen, R. G.; Tasumi, M.; Trezza, R. Satellite-based energy balance for mapping evapotranspiration with internalized calibration (METRIC) - Model. Journal of Irrigation and Drainage Engineering, v.133, p.380-394, 2007b.

Allen, R. G.; Tasumi, M.; Trezza, R. SEBAL (surface energy balance algorithms for land): Advanced training and users manual, version 1.0. Idaho: Implementation, 2002. 97p.

Bastiaanssen, W. G. M. SEBAL-based sensible and latent heat fluxes in the irrigated Gediz Basin, Turkey. Journal of Hidrology, v.229, p.87-100, 2000.

Boegh, E.; Soegaard, H.; Thomsem, A. Evaluating evapotranspiration rates and surface conditions using Landsat TM to estimate atmospheric resistance and surface resistance. Remote Sensing of Environmental, v.79, p.329-343, 2002.

Cabral, O. M. R.; Rocha, H. R.; Ligo, M. A. V.; Brunini, O.; Dias, M. A. F. S. Fluxos turbulentos de calor sensível, vapor d'água e $\mathrm{CO}_{2}$ sobre plantação de cana-de-açúcar (Saccharum sp.) em Sertãozinho-SP. Revista Brasileira de Meteorologia, v.18, p.61-70, 2003.

Chander, G.; Markham, B. L.; Helder, D. L. Summary of current radiometric calibration coefficients for Landsat MSS, TM,ETM+, and EO-1 ALI sensors. Remote Sensing of Environment, v.113, p.893-903, 2009.

Duffie, J. A.; Beckman, W. Solar engineering of thermal processes. New York: John Wiley, 1991. 109p.

EWRI - Task Committee on Standardization of Reference Evapotranspiration of the Environmental and Water Resources Institute of the ASCE (EWRI). 2005. The ASCE Standardization of Reference Evapotranspiration Equation, The ASCE, Reston, Va. 2005.

Garrison, J. D.; Adler, G. P. Estimation of precipitable water over the United States for application to the division of solar radiation into its direct and diffuse components. Solar Energy, v.44, p.225-241, 1990.

Giongo, P. R.; Moura, G. B. A.; Silva, B. B.; Rocha, H. R.; Medeiros, S. R. R.; Nazareno, A. C. Albedo à superfície a partir de imagens Landsat 5 em áreas de cana-de-açúcar e cerrado. Revista Brasileira de Engenharia Agrícola e Ambiental, v.14, p.279-287, 2010.

Gomes, H. B.; Silva, B. B. da; Cavalcanti, E. P.; Rocha, H. R. Balanço de radiação em diferentes biomas de São Paulo mediante imagens Landsat 5. Geociências, v.28, p.153-164, 2009.

Hafeez M. M.; Chemim, Y.; Van De Giesen, N.; Bouman, B. A. M. Field Evapotranspiration in Central Luzon, Philippines, using Different Sensors: Landsat 7 ETM+, Terra Modis and Aster. In: Simposium On Geospatial Theory, Processing And Applications, 2002, Ottawa. Proceedings...Ottawa: Canadian Institute of Geomatics, 2002. 18p.
Iqbal, M. An introduction to solar radiation. New York: Academic Press, 1983. 212p.

Iziomons, M. G.; Mayer, H. On the variability and modeling of surface albedo and long-wave radiation components. Agricultural and Forest Meteorology, v.111, p.141-152, 2002.

Loarie, S. R.; Lobell, D. B.; Asner, G. P.; Mu, Q.; Field, C. B. Direct impacts on local climate of sugar-cane expansion in Brazil. Natura Climate Change. v1. p.105-109. 2011.

Markham, B. L.; Barker, J. L. Thematic mapper bandpass solar exoatmospherical irradiances. Journal of Remote Sensing, v.8, p.517-523, 1987.

Menezes, S. J. M. C.; Sediyama, G. C.; Soares, V. P.; Gleriani, J. M.; Andrade, R. G. Evapotranspiração regional utilizando o SEBAL em condições de relevo plano e montanhoso. Engenharia na Agricultura, v.17, p.491-503, 2009.

Moreira, L. C. J.; Durand, B. J.; Teixeira, A. S.; Andrade, E. M. Variabilidade local e regional da evapotranspiração estimada pelo algoritmo sebal. Engenharia Agrícola, v.30, p.1148-1159, 2010.

Querino, C. A. S.; Moura, M. A. L.; Lyra, R. F. F.; Mariano, G. L. Avaliação e comparação de Radiação solar Global e albedo com ângulo zenital na região amazônica. Revista Brasileira de Meteorologia, v.21, p.42-49, 2006.

Rodrigues, J. O.; Andrade, E. M.; Teixeira, A. S; Silva, B. B. da. Sazonalidade de variáveis biofísicas em regiões semiáridas pelo emprego do sensoriamento remoto. Engenharia Agrícola, v.29, p.452-465, 2009.

Salati, E. Análise ambiental sintética e qualidade da água do rio Corumbataí (SP) como subsídio para o planejamento regional integrado da bacia de drenagem do rio Corumbataí. 198p. São Carlos: UFSCAR, 1996. Tese Doutorado

Silva, B. B.; Braga, A. C; Braga, C. C. Balanço de radiação no perímetro irrigado São Gonçalo-PB mediante imagens orbitais. Revista Caatinga, v.24, p.145-152, 2011.

Silva, B. B. da; Lopes, G. M.; Azevedo, P. V. de. Determinação do albedo de áreas irrigadas com base em imagens Landsat 5 TM. Revista Brasileira de Agrometeorologia, v.13, p.201211, 2005.

Silva, B. B. da; Mendonça, R. R. O.; Silva, S. T. A.; Ferreira, R. C. Mapeamento do albedo de áreas heterogêneas do estado do Ceará com imagens TM - LANDSAT 5. Revista de Geografia, v.25, p.24-41, 2008.

Souza, A. P.; Escobedo, E. F.; Dal Pai, A.; Gomes, E. N. Estimativas das componentes da radiação solar incidente em superfícies inclinadas baseadas na radiação global horizontal. Revista Brasileira de Engenharia Agrícola e Ambiental, v.15, p.277-288, 2011.

SRTM - Shuttle Radar Topography Mission. Disponível em: $<$ http://srtm.csi.cgiar.org/SELECTION/listImages.asp >. 28 Out. 2009.

Tasumi, M.; Allen, R. G.; Trezza, R. At-surface reflectance and Albedo from satellite for operational calculation of land surface energy balance. Journal of Hydrologic Engineering, v.13, p.51-63, 2008. 